\title{
Psicoterapia con sordos. Reflexiones para despatologizar la práctica clínica
}

\author{
Psychotherapy with the Deaf. Reflections to Depathologize \\ Clinical Practice
}
Psicoterapia com surdos. Reflexões para despatologizar a prática clínica

\section{Marcelo Salamanca Salucci*}

\begin{abstract}
RESUMEN
Trabajar con consultantes sordos por veinte años ha implicado ejercitar la auto observación constante, incorporar una actitud crítica hacia los prejuicios existentes en las culturas mayoritarias y aumentar la sensibilidad respecto de la diversidad. Elementos mínimos necesarios para el operar clínico con grupos segregados y para la comprensión de los efectos que generan las diferencias de poder sobre el desarrollo psíquico y emocional.

Descolonizar la psicoterapia con personas sordas es también despatologizar a una minoría sociolingüística y cultural oprimida, sometida históricamente a prácticas de exclusión y a estereotipos atribuidos por una cultura mayoritaria y dominante bajo la etiqueta del audismo.
\end{abstract}

\begin{abstract}
Working with deaf patients over twenty years has involved the exercise of a constant self-observation, incorporating a critical attitude towards the existing prejudices in majority-focused cultures, and raising sensitivity to diversity. These are the minimum elements needed to work with marginalized groups and to understand the effects created by differences in power over their mental and emotional development.
\end{abstract}

Palabras clave: cultura sorda; descolonización; audismo; psicoterapia.
Key words: deaf culture; decolonization; audism; psychotherapy

\footnotetext{
* Académico Adjunto Universidad Católica Silva Henríquez.Psicologo Clínico. Magister en Inclusión de Personas en Situación de Discapacidad. Magister en Psicología Clínica Sistémica. Areas Especialización: Psicología Clínica Sistémica para Personas Sordas y familias mixtas. Contacto: 1salamancas@ucsh.cl
} 
To decolonize psychotherapy with the deaf also means to depathologize a sociolinguistic and culturally oppressed minority, that has been historically subjected to exclusion and stereotyping by a dominating cultural majority, under the label of audism.

\section{Resumo}

Trabalhar com pacientes surdos por vinte anos implicou um exercício de auto-observação constante, incorporando uma atitude crítica em relação aos preconceitos existentes nas principais culturas e aumentando a sensibilidade no que se refere à diversidade. Elementos mínimos necessários para o exercício da

Palavras-chave: cultura surda; descolonização; audismo; psicoterapia. prática clínica com grupos segregados e para a compreensão dos efeitos que as diferenças de poder geram no desenvolvimento emocional e psíquico.

Descolonizar a psicoterapia com pessoas surdas é também despatologizar uma minoria oprimida tanto no aspecto sociolinguístico quanto cultural e historicamente sujeita a práticas de exclusão e estereótipos atribuídos por uma cultura majoritária e dominante sob o rótulo do audismo. 


\section{Introduccion}

En el ejercicio de la psicología clínica desde hace ya veinte años con personas sordas, el acto de desconstrucción y co-construcción epistémica y paradigmática, realizado principalmente a través de la auto observación, ha debido ser una práctica cotidiana en pos de una mejor comprensión de sus problemáticas comunes y bagaje cultural, lo cual ha favorecido la alianza terapéutica con estos consultantes y ha permitido trabajar la autoconciencia respecto de la propia posición frente a ellas y ellos.

Como plantea Falicov (2011), en las nuevas formas de terapia familiar, en las cuales la consideración de las diferencias se encuentra en el núcleo del principio de respeto terapéutico, vemos a todas las familias de manera diferente, todas organizadas de manera única y necesitadas de una descripción más que de una categorización para poder ser entendidas (Anderson y Goolishiam, 1992).

En este sentido, la discusión respecto de la posición que debe adoptar la psicología frente a la diversidad cultural no es nueva y de ella se ha levantado una serie de voces centrando su atención en uno u otro elemento, adoptando o complementando miradas desde y con otras disciplinas, y generando propuestas en las que esta diversidad cultural surge como una noción central impactando a la psicología y, por supuesto, a la forma de hacer psicoterapia.

De esta discusión surge la denominada "psicología cultural", entendida como aquella que se encarga de estudiar el modo en que el comportamiento, las costumbres y la percepción sobre determinados grupos humanos son influenciados por diversos elementos culturales.

\section{Cultura sorda}

Desde esta rama de la psicología, Shweder (1991) entiende que la cultura es una serie de tradiciones y prácticas sociales que regula, expresa y transforma la psiquis humana. A esto Matsumoto (2000) agrega que la cultura actúa como un filtro, no solamente cuando percibimos cosas sino también cuando pensamos e interpretamos eventos, y que, puesto que todos existimos dentro de nuestras propias culturas y en nuestros propios contextos culturales, tendemos a ver las cosas a través de esos filtros. En este sentido, mente y cultura son indivisibles y 
mutuamente constitutivas, por tanto, las personas son modeladas por la cultura y viceversa.

Compartir el idioma, sistema de significados o de creencias, visión del mundo o experiencias, usualmente otorga un sentido de familiaridad y comunidad a las personas que comparten la misma cultura, pero a la vez existen inconsistencias, diversidades, y novedades junto a estas dimensiones (Falicov, 2011).

Ahora, en el trabajo con la diversidad, una de las culturas con las que el terapeuta se puede encontrar es la que posee, para un grupo importante de personas al menos, la comunidad sorda, entendida ésta por Lee Baker-Shenk y Padden (1978) como aquel grupo humano que abarca a personas sordas o con algún tipo de pérdida auditiva, que comparten una lengua, experiencias y valores, y una forma común de interactuar entre ellas y con las personas oyentes (Ladd, 2003).

Mucho antes que ellos, Croneberg (Stokoe, Dorothy \& Croneberg, 1965) acuñó el concepto de "cultura sorda", el cual, desde que usó por primera vez hasta nuestros días, se ha divulgado en un sinfín de contextos, siendo enriquecido cada vez por personas sordas y oyentes provenientes de las más diversas áreas.

Para Padden y Humphries (2005) hoy existe consenso en entender a la cultura sorda como un conjunto de creencias sociales, comportamientos, arte, tradiciones, historia y valores, compartidos por una comunidad influenciada por la sordera y que utiliza la lengua de señas como su principal medio de comunicación. Para Leigh (2010) esta comunidad está constituida por una amplia diversidad de comunidades sociales, políticas, culturales o religiosas, que involucran tanto a personas sordas como oyentes conectadas por una forma de comunicación visogestual: la lengua de señas.

Por su parte, Ladd (2003) afirma que la construcción de una teoría cultural es una forma de resistencia, y reconocer la cultura sorda y las visiones subalternas sordas existentes dentro del ámbito académico es un paso fundamental para crear una base que desarticule el discurso oralista, llegando así a personas legas y obteniendo el apoyo que se requiere para derrocar el colonialismo, en este caso el oyentismo, que ha sometido a las personas sordas en todas las formas posibles. 
$\mathrm{Al}$ respecto Di Nicola (como se citó en Andolfi, 2009) señala que la cultura es el cierre de las relaciones humanas, el camino para comprender la mente, el sí mismo y la identidad. Algo que para Matsumoto (2000) dificultará la comprensión del comportamiento de otros, ante la imposibilidad de separarse de los propios contextos y sesgos culturales, y podría ser la base del etnocentrismo.

Sin embargo, el propio Matsumoto (2000) agrega que, puesto que esto es una consecuencia propia del proceso de socialización y enculturación, todas las personas son etnocéntricas en cierta medida y que ver e interpretar las cosas a través de los propios filtros culturales no es necesariamente perjudicial ni reviste en sí un peligro, sino más bien el no reconocerlo ni estar consciente de su existencia.

En ese sentido, Falicov (2011) agrega que sobre las identidades culturales también influyen los conceptos provenientes de los discursos dominantes y que tener en cuenta estas influencias, y la multitud de mezclas resultantes, ayuda a evitar pensar a las culturas como si fueran estáticas.

\section{Perspectiva sistémico-cultural}

Vivir en una sociedad globalizada, donde coexisten cada vez más grupos humanos y se diversifican las culturas, implica la necesidad de que la psicoterapia cuestione la universalidad de sus teorías y técnicas, e incorpore un pensamiento sistémico multicultural que considere las múltiples pertenencias a sistemas o contextos que puede llegar a tener un sistema consultante. No obstante, se debe evitar el riesgo de que esto se transforme en el estereotipo de ciertos grupos culturales o el no reconocimiento de la amplitud de variaciones que puede existir dentro de un mismo colectivo humano.

Puesto que aún no existe consenso en todas las disciplinas ni en todas las latitudes respecto de lo que interculturalidad, multiculturalidad, pluralidad cultural o transculturalidad puedan implicar realmente, es común ver que estos conceptos sean tratados como similares, generando así un grado mayor de confusión. Ante esto, Cervantes (2006) señala que un considerable número de disciplinas, tales como la historia, la etnología, la antropología, la filosofía, la educación, la psicología y la política, entre muchas otras, han heredado una multiplicidad 
de nociones que incrementan el menú de términos que no solo no se puede utilizar como sinónimos, sino que hacen más denso el estudio de sus relaciones y distinciones.

Gergen y McNamee (1996) refieren que esto, entre otras muchas variables, ha generado que algunos autores argumenten que el movimiento hacia la psicología cultural debe ser en realidad un movimiento hacia una de enfoque multicultural. Una que incorpore las psicologías particulares de la diversidad de culturas existentes alrededor del mundo y que no pueden ser asimilables a una sola psicología.

Es así como el movimiento multicultural de los años 90 ha revitalizado estas inquietudes, en el marco de aquellas nuevas epistemologías posmodernas que enfatizan una visión social construccionista, tienen una orientación basada en las fortalezas y un compromiso colaborativo con los clientes (Falicov, 2011).

En este sentido, se plantea que el multiculturalismo ha venido a desafiar, desde hace un buen tiempo, lo que las escuelas tradicionales del pensamiento psicológico han considerado universal.

Pese a esto, aún se debate la pertinencia de que el psicoterapeuta conozca los elementos culturales de un determinado grupo humano, puesto que ello implicaría el riesgo de entrar al espacio terapéutico con prejuicios o presuposiciones basados en estereotipos respecto de este grupo y porque la información culturalmente relevante podría ser a su vez proporcionada por cada consultante (Rincón, 2019).

Sin embargo, para aquellos psicoterapeutas que trabajan con consultantes sordos y que desconocen los elementos de su cultura, existe el riesgo de caer en un entrampe cultural del cual solo se puede salir en el acto de deconstruir los discursos colonizadores, en este caso oyentizadores, a los que ha sido expuestos; de descolononizar sus prácticas, en este caso desoyentizar, y de co-construir nuevos relatos que revelen y reviertan situaciones y actos institucionales, culturales y epistemológicos afectados por esta visión opresiva-médico-clínica, y otros mecanismos de subordinación y poder que han dominado los discursos vinculados a las personas sordas, principalmente en la educación y la psicología.

En este sentido, deconstruir implicará abandonar los juicios absolutos sobre lo que son o no son las cosas, y mantenerse alejados y 
escépticos respecto de lo que se cree pueda ser la verdad cerca de las personas sordas y la sordera (Salamanca, 2019).

Este acto de deconstrucción/descolonización es, para algunos, una cuestión de justicia social y, como tal, asocia los problemas de salud mental a experiencias de opresión social y busca otorgar poder a las familias en sus interacciones con sistemas más grandes y con otros discursos culturales, incluyendo aquellos presentes en el campo de la psicoterapia (Korin, como se citó en Falicov, 2011; Hardy \& Lazzloffy, 1994).

En el terreno de la clínica, esta postura acerca de la justicia social dirige la atención hacia las condiciones de vida, relaciones de poder y prejuicios que limitan las oportunidades sociales y económicas, promueven el racismo internalizado y afectan el desarrollo psicológico y la salud mental de quienes son pobres o marginados (Falicov, 2011).

\section{Psicoterapia con consultantes sordos}

Vernon (como se citó en Leigh, 1999) refiere que, históricamente, han sido profesores, entrenadores deportivos, miembros del clero y trabajadores sociales los más involucrados en la vida de las personas sordas; y que por años estas personas fueron quienes llenaron el vacío de la salud mental de los sordos, proveyéndoles ayuda con cualquier tipo de asistencia sencilla, consejos, directrices, instrucciones, charlas o amonestaciones.

Para Sussman \& Brauer (como se citó en Leigh, 1999) ha sido por causa de la limitada percepción respecto de las capacidades de los individuos sordos que la psicoterapia para ellos se ha tornado engorrosa y se ha privilegiado enfoques directivos, obsoletos y anacrónicos.

Afortunadamente, hoy son cada vez más los psicólogos interesados en trabajar con personas sordas, ya no exclusivamente en contextos educativos, donde principalmente se dedican a la evaluación de estudiantes sordos, si no desde un ámbito más clínico.

Sin embargo, esto significará que, a la par de la formación teórica y técnica, los terapeutas requerirán de un trabajo constante de autoconocimiento en su operar, que les permitirá tener mayor conciencia del filtro a través del cual se interpreta la realidad, puesto que, tal como señala Okun (como se citó en Moreno, 2014), al desarrollar esta concien- 
cia social, cultural y de género, los terapeutas se harán más conscientes de los estereotipos o prejuicios que puedan estar condicionando su percepción sobre lo que es normal o adecuado.

Frente a esto, Glickman y Gulati (2003) plantean que los profesionales de la salud mental, frente al consultante sordo, podrán situarse dentro en un continuo de cinco parámetros, que va desde el rechazo cultural hasta la promoción cultural.

Para él, en el primer nivel de este continuo están quienes, influenciados por una visión médico-clínica, desconocen que las personas sordas posean una cultura propia, consideran la sordera como una enfermedad que debe ser curada, ven a las personas sordas con cierta lástima o niegan la importancia de la lengua de señas y del pertenecer a una comunidad sorda; luego están aquellos profesionales que son insensibles culturalmente, y que por simple ignorancia, aun cuando tienen toda la voluntad, atienden a consultantes sordos y a sus familias, y cometen una serie de errores por su desconocimiento respecto de la cultura sorda y la lengua de señas; a ellos les sigue el nivel conformado por profesionales sensibles culturalmente, quienes reconocen algunas de las necesidades de las personas sordas y pueden tener una actitud más abierta en la interacción con ellas.

En los dos últimos niveles, denominados por Glickman y Gulati (2003) como "afirmativo culturalmente" y "promotor de la cultura sorda", los autores ubican a aquellos profesionales que tienen conocimientos y habilidades específicas para el trabajo con esta comunidad, y reflexionan en torno a ello constantemente. Sin embargo, aun cuando el autor destaca el hecho de que estos profesionales tengan las competencias culturales para el ejercicio clínico, las habilidades y herramientas necesarias para poder comunicarse de manera fluida con sus consultantes sordos y la capacidad de reafirmarlos culturalmente, señala que lo óptimo es que los profesionales de la salud mental no se dediquen a la promoción cultural, puesto que, además de irrespetuoso con ciertos consultantes, puede poner en riesgo la co-construcción del setting con aquellos que no han desarrollado la identidad cultural sorda.

Como se mencionó, para Glickman y Gulati (2003) el profesional de la salud mental que trabaja con personas sordas se ubicará en alguno 
de los cinco estadios, dependiendo de su experiencia, cercanía y sensibilidad hacia la comunidad sorda y su cultura. En este sentido, de la conjunción de estos elementos dependerá en gran medida el éxito o fracaso de su intervención.

De lo anterior se puede desprender que el logro de los objetivos terapéuticos propuestos por el terapeuta estará estrechamente relacionado con su acervo cultural respecto de la comunidad sorda, lo cual puede ser paradojal, si se considera que la gran mayoría de los psicólogos clínicos que en Latinoamérica trabajan con consultantes sordos son oyentes (Salamanca, 2013) y probablemente podrían ser clasificados mayoritariamente dentro de los primeros niveles del continuo propuesto por Glickman y Gulati (2003).

En el caso de psicoterapeutas oyentes que se adscriban a la cultura sorda, identificándose a sí mismos como miembros de esta comunidad y siendo aceptados por los demás como parte de ésta, Ladd (2003) habla de una sordera actitudinal, algo que se evidencia, por ejemplo, en el caso de aquellos psicólogos que tienen algún tipo de vínculo sanguíneo (filial, parental, fraternal) o por afinidad (conyugal) con personas sordas.

A lo señalado por Ladd (2003), Salamanca (2019) agrega la importancia que reviste el hecho de que el terapeuta sea además una persona bilingüe, es decir fluente en lengua de señas; que reconozca y valore la sordedad (deafhood) planteada por el mismo Ladd (2003); que considere, tal como lo propone Bauman (2004), la ganancia que puede significar el ser sordo (deaf gain) y el aporte que esta comunidad ha hecho a la sociedad; y que sepa de la opresión a la que ha sido sometida la comunidad sorda, principalmente por una comunidad oyente mayoritaria, expresada en múltiples formas agrupadas en lo que Humphries (1975), en una primera instancia, y posteriormente Lane (1992) denominaron "audismo" (audism).

Por otra parte, un psicoterapeuta que se propone trabajar con personas sordas debe tener una actitud de apertura hacia el intercambio cultural y valorar la riqueza de la diversidad, entendida ésta como un elemento constitutivo de las relaciones humanas, a través de la cual podemos aumentar el conocimiento de la realidad que nos rodea (Bateson, como se citó en Andolfi, 2009). 
Acorde con esto, Salamanca (2019) propone estar consciente de los filtros culturales con que se observa y que surgen en el terapeuta y en cada uno de los miembros del sistema consultante, en este caso en particular en miembros de una minoría sociolingüística, y entender el rechazo que puede provocar en las personas sordas el trabajar con un terapeuta oyente.

En este sentido, Harvey (2013) señala la importancia de preguntarse constantemente, como terapeutas, sobre la motivación de trabajar con personas sordas y estar atentos a que esta no responda a patrones opresivos, colonizadores, oyentistas o audistas, ni que menoscaben la autonomía e identidad sorda.

Para Glickman y Harvey (1996), una terapia efectiva constituye una práctica culturalmente informada que se piensa y se cuestiona la realidad de la estructura social, la cultura, el poder y la opresión, y que involucra intervenciones culturalmente relevantes; una que busca propiciar el empoderamiento de las personas consultantes y de sus comunidades, que establece conexiones entre la experiencia individual y colectiva, y que mantiene un balance entre las consideraciones clínicas y las consideraciones culturales (Glickman y Harvey, como se citó en Rincón 2019).

Para Gergen y McNamee (1996), de igual forma que el construccionismo, este modelo sitúa el origen de la acción humana en las relaciones, remitiendo la comprensión del funcionamiento individual al intercambio comunitario entre individuos que comparten un determinado contexto cultural, en el cual, a través de la permanente conversación con sus cercanos, se desarrollará un sentimiento de identidad o voz interior.

Por tanto, puesto que el yo no está dentro de la persona, esa identidad deja de ser individual y se transforma en algo construido a partir de interacciones sociales - paradojalmente restringidas en la mayoría de las personas sordas-, las que posibilitan la construcción de una idea de personalidad.

Esto permite al terapeuta desviar la responsabilidad exclusiva de la enfermedad desde el consultante sordo hacia sus grupos de pertenencia y realizar intervenciones que apunten a lograr que estas personas puedan asumir parte activa de actos y situaciones reivindicativas, en- 
tendiendo ahora los problemas, tal como lo plantean Anderson y Goolishian (1996), como acciones que expresan las narraciones humanas, que existen en el lenguaje y que son propios del contexto narrativo del que derivan su significado y sobre el cual se debe crear dialógicamente una nueva narración.

En este sentido, Gergen y McNamee (1996) plantean que, a medida que el foco se desplaza a la construcción lingüística de la realidad, los problemas y las enfermedades pierden su privilegio ontológico, ya que no son independientes sino construcciones culturales. Por tanto, no habrá problemas, más allá de la forma en que una cultura los constituya como tales.

\section{Referencias}

Anderson, H. y Goolishian, H. (1996). El experto es el cliente: la ignorancia como enfoque terapéutico. En: S. McNamee y K. Gergen (coords.). La terapia como construcción social (pp. 4559). Barcelona: Paidós.

Andolfi, M. (2009). La psicoterapia como viaje transcultural. Psicoperspectivas, VIII(1), 6-44. Recuperado de http://www.psicoperspectivas.cl

Bauman, L. (2004) Audism: Exploring the Metaphysics of Oppression. Journal of Deaf Studies and Deaf Education, 9 (2), 239246. DOI:10.1093/deafed/enh025.

Cervantes, C. (2006) Diversidad cultural y nociones relacionadas: un análisis conceptual. En R. Mejía-Arauz, S. Frisancho, Investigar la diversidad cultural. Teoría, conceptos y métodos de investigación para la educación y el desarrollo. ITESO/Universidad de Colima/IBERO.

Falicov, C. (2011). La cultura en la Terapia Familiar. Nuevas variantes de un tema fundamental. Redes: revista de psicoterapia relacional e intervenciones sociales, 25, 43-68.

Gergen, K. y McNamee, S. (1996) La terapia como construcción social. Editorial Paidós.

Glickman, N. \& Harvey, M. (1996). Culturally affirmative psychotherapy with deaf persons. Lawrence Erlbaum Associates, Publishers.

Glickman, N. \& Gulati, S. (2003). Mental health care of deaf people. A culturally affirmative approach. Lawrence Erlbaum Associates, Publishers. 
Hardy, K. \& Laszloffy, T. (1994). Deconstructing race in family theraphy. Journal of Feminist Family Theraphy, 5(3/4), 5-33.

Harvey, M. (2013). Psychotherapy with deaf and hard of hearing persons: a systemic model. Routledge Published.

Humphries, T. (1975). Audism: The Making of a Word. Unpublished paper.

Ladd, P. (2003). Understanding Deaf Culture: In Search of Deafhood. Clevedon, England: Multilingual Matters.

Lane, H. 1992. The Mask of Benevolence: Disabling the Deaf Community. New York: Knopf Print.

Lee Baker-Shenk, C.; Padden, C. (1978). American Sign Language: A Look at Its History, Structure, and Community T. J. Publishers.

Leigh, I. (2010). Psychotherapy with deaffrom diverse groups. Washington: Gallaudet University Press.

Matsumoto, D. (2000). Culture and psychology: People around the world (2nd ed.). Wadsworth/Thomson Learning.

Moreno, A. (2014). Manual de terapia sistémica. Principios y herramientas e intervención. Editorial Desclee.

Padden, C.; Humphries, T. (2005). Inside Deaf Culture. Cambridge, MA: Harvard University Press.

Rincón, Edna. 2019. Psicoterapia culturalmente afirmativa: una investigación implicada con la Comunidad Sorda. Tesis para optar al título de Magíster en Discapacidad e Inclusión Social. Universidad Nacional de Colombia, Facultad de Medicina, Bogotá, Colombia.

Salamanca, M. (2013) Terapia sistémica para familias mixtas. En M. Schorn (Presidencia), Salud Mental y Sordera. III Congreso internacional, V Congreso latinoamericano y VI Congreso Nacional de Salud Mental y Sordera. Buenos Aires, Argentina.

Salamanca, M. (2019). Intervenciones clínicas para consultantes sordos y sus familias. En R. Claros-Kartchner (Presidencia), Educación de estudiantes sordos. III Simposio para la educación de estudiantes sordos. Santo Domingo, República Dominicana.

Shweder, R. (1991). Thinking through cultures: Expeditions in cultural psychology. Harvard University Press. 
Stokoe, W., Dorothy, C. \& Croneberg, C. (1965). A dictionary of American sign languages on linguistic principles. Washington, D.C.: Gallaudet College Press. 